\title{
Perceived parental affectionless control is associated with high neuroticism
}

\author{
This article was published in the following Dove Press journal: \\ Neuropsychiatric Disease and Treatment \\ 18 April 2017 \\ Number of times this article has been viewed
}

\author{
Nana Takahashi \\ Akihito Suzuki \\ Yoshihiko Matsumoto \\ Toshinori Shirata \\ Koichi Otani \\ Department of Psychiatry, Yamagata \\ University School of Medicine, \\ Yamagata, Japan
}

\begin{abstract}
Objective: Depressed patients are prone to perceive that they were exposed to affectionless control by parents. Meanwhile, high neuroticism is a well-established risk factor for developing depression. Therefore, this study examined whether perceived parental affectionless control is associated with high neuroticism.
\end{abstract}

Methods: The subjects were 664 healthy Japanese volunteers. Perceived parental care and protection were assessed by the Parental Bonding Instrument. Parental rearing was categorized into either optimal parenting (high care/low protection) or three dysfunctional parenting styles including affectionless control (low care/high protection). Neuroticism was evaluated by the NEO Personality Inventory-Revised.

Results: The subjects with paternal affectionless control had higher neuroticism scores than those with paternal optimal parenting. Similar tendency was observed in maternal rearing. Neuroticism scores increased in a stepwise manner with respect to the increase in the number of parents with affectionless control.

Conclusion: The present study shows that perceived parental affectionless control is associated with high neuroticism, suggesting that this parental style increases neuroticism in recipients.

Keywords: parenting, attachment, personality, vulnerability, depression, PBI, NEO PI-R

\section{Introduction}

According to Bowlby's attachment theory, ${ }^{1}$ pathogenic parents who are unresponsive to a child's desire for care and/or do not allow the child's progressive independence create anxious attachment in the child. The anxiously attached child grows up to be anxious, insecure, over-dependent, or immature and is predisposed to develop psychiatric disorders such as depression under stress. ${ }^{1}$ Under the influence of attachment theory, Parker et $\mathrm{al}^{2}$ developed the Parental Bonding Instrument (PBI) to assess perceived parental care and protection. By using these care and protection scores, they defined four parenting styles, namely, optimal parenting (high care/low protection), affectionate constraint (high care/high protection), neglectful parenting (low care/ low protection), and affectionless control (low care/high protection). Subsequent studies $^{3-6}$ show that depressed patients are prone to perceive that they were exposed to affectionless control parenting.

Neuroticism is a tendency to cope poorly with stress and to experience negative feelings of sadness, anxiety, and irritability. ${ }^{7-10}$ Behavioral genetic studies suggest that roughly $40 \%$ of the variability in this personality trait is attributed to genetic factors and the rest to environmental factors. ${ }^{9}$ Extensive reviews consistently show that high neuroticism is a risk factor for developing depression. ${ }^{8,10,11}$ A complex interplay between this personality trait and stressful life events, that is, a diathesis-stress scenario,
Correspondence: Yoshihiko Matsumoto Department of Psychiatry, Yamagata University School of Medicine,

2-2-2 lidanishi, Yamagata City,

Yamagata 990-9585, Japan

Tel +8I 236285322

Fax +8I 236285325

Email matsumotoyo@mvc.biglobe.ne.jp 
has been proposed ${ }^{8,10}$ as a possible mechanism underlying this neuroticism-depression association.

Our previous studies suggest that affectionless control parenting increases personality vulnerabilities to depression such as harm avoidance in psychobiological model of personality, ${ }^{12}$ interpersonal sensitivity within the frame of attachment theory, ${ }^{13}$ and dysfunctional attitudes in cognitive model of depression. ${ }^{14}$ These findings led to the hypothesis that this parenting style also increases neuroticism. To test this hypothesis, the present study examined whether perceived parental affectionless control is associated with high neuroticism. As mentioned earlier, the present study was based on the diathesis-stress model of depression, which regards high neuroticism as a diathesis. ${ }^{8,10}$ Meanwhile, it is known that reports of personality traits such as neuroticism are influenced by mood states associated with psychiatric disorders. ${ }^{8}$ Therefore, a nonclinical sample with arbitrary levels of neuroticism but without major psychiatric disorders was used so far.

\section{Methods}

\section{Subjects}

First, 750 Japanese without serious physical diseases were recruited from medical students and medical staffs in Yamagata Prefecture. Screening for psychiatric disorders was performed by a brief interview and by using a questionnaire on present or past psychiatric disorders. For the interview, six items were selected from the Structured Clinical Interview for DSM-IV Axis I Disorders, ${ }^{15}$ that is, A1 for major depressive episode, A16 for manic episode, B1 for delusions, B6 for hallucinations, E2 for alcohol abuse, and F68 for anxiety disorders. Of the 750 cases, 27 had psychiatric disorders, 12 had parents divorced or deceased before the age of 16 years, and 47 had missing data. Hence, these 86 cases were excluded, and the remaining 664 cases were used for analyses. Of them, 432 were males, and 232 were females. The mean \pm standard deviation of age was $23.4 \pm 2.2$ years. The study protocol was approved by the Ethics Committee of Yamagata University School of Medicine, and all the subjects gave written informed consent to participate.

\section{Assessment of perceived parenting styles}

Perceived parental care and protection during the first 16 years were assessed by the Japanese version ${ }^{16}$ of the PBI, through which reliability and validity have been confirmed. The number of items of the care subscale and protection subscale is 12 and 13, respectively. Participants evaluate the extent to which their parents match each description on a 4-point scale ranging from 0 that represents "very unlike" to 3 that represents "very like." In these subjects, Cronbach's $\alpha$ for father care, father protection, mother care, and mother protection subscales were $0.92,0.85,0.90$, and 0.85 , respectively. Care with a care subscale score equal to or lower than the median was regarded as low care, whereas the other was regarded as high care. The same standard was used to determine the levels of protection. Subsequently, paternal and maternal rearing practices were classified into four types as defined by Parker et al, ${ }^{2}$ namely, optimal parenting (high care/low protection), affectionate constraint (high care/high protection), neglectful parenting (low care/low protection), and affectionless control (low care/high protection).

\section{Assessment of neuroticism}

Neuroticism was evaluated by the Japanese version ${ }^{17}$ of the NEO Personality Inventory-Revised (NEO PI-R). ${ }^{18}$ This Japanese version has high reliability and validity. ${ }^{17}$ The neuroticism dimension scale of the NEO PI-R has six facet scales, each with eight items. Participants assess the degree to which they correspond to each phrase on a scale with 5 anchor points ranging from "strongly disagree" to "strongly agree." In the present sample, Cronbach's $\alpha$ for the neuroticism dimension scale was 0.84 .

\section{Statistical analyses}

Statistical analyses were performed by one-way and two-way analysis of covariance (ANCOVA) with sex as a covariate followed by least significant difference test by using SPSS $14.0 \mathrm{~J}$ for Windows. $P$-value $<0.05$ was considered statistically significant.

\section{Results}

Table 1 shows the effects of perceived parenting styles on neuroticism scores. Paternal styles (partial $\eta^{2}=0.024$ ) and maternal styles (partial $\eta^{2}=0.016$ ) had significant effects on neuroticism scores, with no significant interaction effect between them. The subjects with paternal affectionless control showed higher neuroticism scores than those with paternal optimal parenting. Similarly, subjects with maternal affectionless control showed higher neuroticism scores than those with maternal optimal parenting. Affectionate constraint by mothers but not by fathers was related to higher neuroticism scores. Neglectful parenting by fathers but not by mothers was related to higher neuroticism scores.

Table 2 shows the effect of the number of parents with perceived affectionless control on neuroticism scores. The number of parents with affectionless control had a significant effect (partial $\eta^{2}=0.050$ ) on neuroticism scores. Group 2 with two 
Table I Effects of perceived parenting styles on neuroticism scores

\begin{tabular}{lll}
\hline Parenting style & N & Neuroticism \\
\hline Father & & \\
Optimal parenting & 207 & $96.5 \pm 20.9$ \\
Affectionate constraint & 115 & $103.0 \pm 19.9$ \\
Neglectful parenting & 133 & $104.4 \pm 22.6^{\mathrm{a}}$ \\
$\quad$ Affectionless control & 209 & $108.6 \pm 21.9^{\mathrm{b}}$ \\
Mother & & \\
Optimal parenting & 222 & $97.6 \pm 21.1$ \\
Affectionate constraint & 77 & $107.7 \pm 22.5^{\mathrm{c}}$ \\
Neglectful parenting & 145 & $100.9 \pm 20.6$ \\
Affectionless control & 220 & $108.2 \pm 21.9^{\mathrm{d}}$ \\
ANCOVA (P) & & \\
Father effect & & 0.001 \\
Mother effect & & 0.015 \\
Interaction effect & & 0.557
\end{tabular}

Notes: The values in the table are expressed as mean \pm SD. P-values of LSD test; ${ }^{\mathrm{a}} 0.034,{ }^{b} 0.000,{ }^{c} 0.013,{ }^{d} 0.018$, compared with optimal parenting.

Abbreviations: ANCOVA, two-way analysis of covariance; SD, standard deviation; LSD, least significant difference.

parents of affectionless control and Group 1 with one parent of affectionless control had higher neuroticism scores than Group 0 with no parent of affectionless control. The number of parents with perceived affectionate constraint $(P=0.674)$ and that of parents with perceived neglectful parenting $(P=0.284)$ had no significant effects on neuroticism scores.

\section{Discussion}

In the first analysis, perceived affectionless control from fathers and that from mothers were both associated with high neuroticism. In the second analysis, neuroticism increased in a stepwise manner according to the increase in the number of parents with this rearing style. On the contrary, the association with high neuroticism of affectionate constraint was confined to maternal rearing and that of neglectful parenting was confined to paternal rearing. Also, the number of parents with these rearing styles had no significant effects on neuroticism. These results suggest that among the three

Table 2 Effect of number of parents with perceived affectionless control on neuroticism scores

\begin{tabular}{lll}
\hline $\begin{array}{l}\text { Number of affectionless } \\
\text { control parents }\end{array}$ & $\mathbf{N}$ & Neuroticism \\
\hline Group 0 & 371 & $99.4 \pm 21.1$ \\
Group I & 157 & $105.6 \pm 22.5^{\mathrm{a}}$ \\
Group 2 & 136 & $110.0 \pm 21.4^{\mathrm{b}}$ \\
ANCOVA $(P)$ & & 0.000 \\
\hline
\end{tabular}

Notes: The values in the table are expressed as mean \pm SD. Group 0: no parent with affectionless control, Group I: one parent with affectionless control, Group 2: two parents with affectionless control. $P$-values of LSD test: ${ }^{a} 0.000$, ${ }^{b} 0.000$, compared with optimal parenting.

Abbreviations: ANCOVA, one-way analysis of covariance; SD, standard deviation; LSD, least significant difference. anomalous parenting styles, affectionless control is most strongly connected with high neuroticism in recipients. Therefore, we add high neuroticism to the list of personality vulnerabilities to depression, correlated with perceived affectionless control parenting, that is, high harm avoidance, ${ }^{12}$ interpersonal sensitivity, ${ }^{13}$ and dysfunctional attitudes. ${ }^{14}$

Importantly, Parker ${ }^{5}$ reported that perceived parental characteristics assessed by the PBI reflect the actual parental characteristics assessed by significant others with acceptable validity. Therefore, the strong connection between perceived parental affectionless control and high neuroticism observed in the present study suggests that this parental style increases neuroticism in recipients. As affectionless control is a combination of the pathogenic parenting practices proposed by Bowlby, ${ }^{1}$ it is likely that this parental style induces anxious attachment. Therefore, it is suggested that high neuroticism arises as an expression of anxious attachment. This view is supported by the substantial overlap between the personality characteristics of high scorers of neuroticism, that is, anxious, impatient, pessimistic, timid, self-centered, and dependent, ${ }^{7}$ and those of anxiously attached individuals mentioned earlier. ${ }^{1}$ It is also supported by the correlations of neuroticism with attachment anxiety and attachment avoidance, ${ }^{19}$ the indices of anxious attachment. Taken together, close interrelations are suggested among affectionless control parenting, anxious attachment, and high neuroticism, all of which are linked to the development of depression.

There are three possible limitations in the present study. First, the reporting of parental affectionless control by high scorers of neuroticism may be partly ascribable to their perceptual bias because of the inherent limitation of the PBI, that is, parental rearing during childhood is recalled in adulthood. Second, children with a specific temperament by nature, for example, high neuroticism, may elicit a specific rearing style from parents, that is, affectionless control. In light of these possibilities, we may have to be more careful in interpreting the causal relationship between affectionless control parenting and high neuroticism. Third, the subjects were all well-educated young Japanese, and because of these specificities, the present results should be extrapolated cautiously to populations with other demographic or ethnic backgrounds.

\section{Conclusion}

The present study shows that perceived parental affectionless control is associated with high neuroticism, suggesting that this parental style increases neuroticism in recipients. 


\section{Acknowledgments}

This study was supported by funding from the Ministry of Education, Culture, Sports, Science and Technology of Japan. This had no effect on this study.

\section{Disclosure}

All authors report no conflicts of interest in this work.

\section{References}

1. Bowlby J. The making and breaking of affectional bonds. I. Aetiology and psychopathology in the light of attachment theory. Br J Psychiatry. 1977;130:201-210.

2. Parker G, Tuping H, Brown LB. A parental bonding instrument. $B r J$ Med Psychol. 1979;52:1-10.

3. Parker G. Parental characteristics in relation to depressive disorders. Br J Psychiatry. 1979;134:138-147.

4. Parker G. Parental "affectionless control" as an antecedent to adult depression. Arch Gen Psychiatry. 1983;40(9):956-960.

5. Parker G. The measurement of pathogenic parental style and its relevance to psychiatric disorder. Soc Psychiatry. 1984;19(2):75-81.

6. Grant K-A, Bautovich A, McMahon C, Reilly N, Leader L, Austin M-P. Parental care and control during childhood: associations with maternal perinatal mood disturbance and parenting stress. Arch Womens Ment Health. 2012;15(4):297-305.

7. McCrae RR, Costa PT. NEO Inventories for the NEO Personality Inventory-3 (NEO-PI-3), NEO Five-Factor-3 (NEO-FFI-3), NEO Personality Inventory-Revised (NEO PI-R), Professional Manual. Lutz, FL: Psychological Assessment Resources; 2010.

8. Klein DN, Kotov R, Bufferd SJ. Personality and depression: explanatory models and review of the evidence. Annu Rev Clin Psychol. 2011; 7:269-295.
9. Cervone DC, Pervin LA. Trait theory: the five-factor model; applications and evaluation of trait approaches to personality. In: Personality: Theory and Research. 12th ed. Cervone D, Pervin LA, eds. Hoboken, NJ: John Wiley \& Sons; 2013:261-300.

10. Ormel J, Jeronimus BF, Kotov R, et al. Neuroticism and common mental disorders: meaning and utility of a complex relationship. Clin Psychol Rev. 2013;33(5):686-697.

11. Enns M, Cox BJ. Personality dimensions and depression: review and commentary. Can J Psychiatry. 1997;42(3):274-284.

12. Otani K, Suzuki A, Oshino S, Ishii G, Matsumoto Y. Effects of the "affectionless control" parenting style on personality traits in healthy subjects. Psychiatry Res. 2009;165(1-2):181-186.

13. Otani K, Suzuki A, Shibuya N, Matsumoto Y, Kamata M. Dysfunctional parenting styles increase interpersonal sensitivity in healthy subjects. J Nerv Ment Dis. 2009;197(12):938-941.

14. Otani K, Suzuki A, Matsumoto Y, Sadahiro R, Enokido M. Affectionless control by the same-sex parents increases dysfunctional attitudes about achievement. Compr Psychiatry. 2014;55(6):1411-1414.

15. First MB, Spitzer RL, Gibbon M, Williams JBM. Structured Clinical Interview for DSM-IV Axis I Disorders - Clinician Version. Washington, DC: American Psychiatric Association; 1997.

16. Ogawa M. [A study of the reliability and validity of the Japanese version of the PBI (Parental Bonding Instrument)]. Seishinkachiryougaku. 1991;6(10):1193-1201. Japanese

17. Shimonaka Y, Nakazato K, Gondo Y, Takayama M. NEO-PI-R, NEOFFI Manual for the Japanese Version. Tokyo: Tokyo Shinri; 1999. Japanese.

18. Costa PT, McCrae RR. NEO-PI-R Professional: Revised NEO Personality and NEO Five-Factor Inventory (NEO-FFI). Odessa, FL: Psychological Assessment Resources; 1992.

19. Mikulincer M, Shaver PR. Attachment bases of psychopathology. In: Attachment in Adulthood: Structure, Dynamics, and Change. Mikulincer M, Shaver PR, eds. New York: Guilford Press; 2007:369-404.
Neuropsychiatric Disease and Treatment

\section{Publish your work in this journal}

Neuropsychiatric Disease and Treatment is an international, peerreviewed journal of clinical therapeutics and pharmacology focusing on concise rapid reporting of clinical or pre-clinical studies on a range of neuropsychiatric and neurological disorders. This journal is indexed on PubMed Central, the 'PsycINFO' database and CAS,

\section{Dovepress}

and is the official journal of The International Neuropsychiatric Association (INA). The manuscript management system is completely online and includes a very quick and fair peer-review system, which is all easy to use. Visit http://www.dovepress.com/testimonials.php to read real quotes from published authors. 